Document downloaded from:

http://hdl.handle.net/10251/104245

This paper must be cited as:

Machado-Olivares, FJ.; Zagrajek, P.; Monsoriu Serra, JA.; Furlan, WD. (2018). Terahertz Sieves. IEEE Transactions on Terahertz Science and Technology. 8(1):140-143. doi:10.1109/TTHZ.2017.2762292

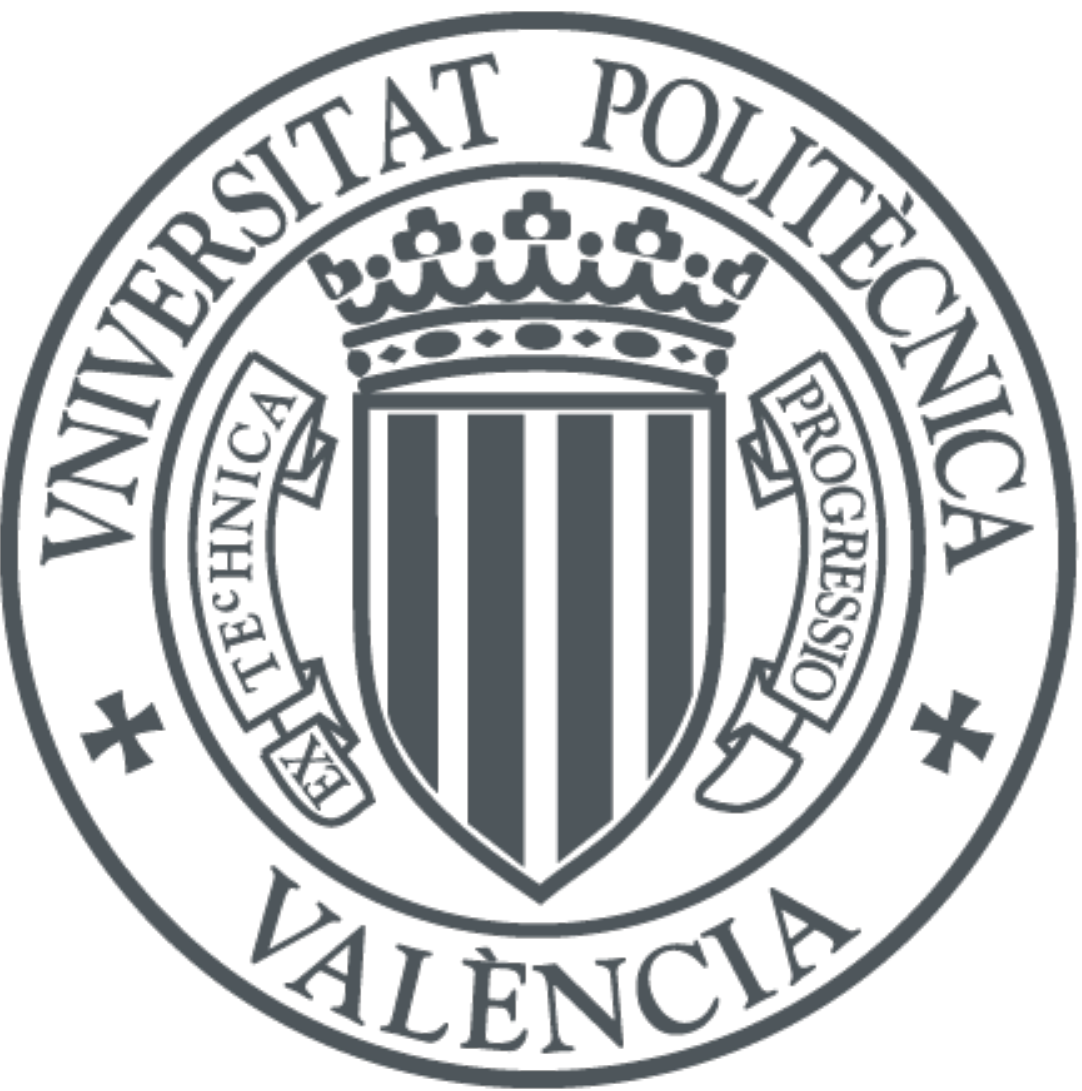

The final publication is available at

https://doi.org/10.1109/TTHZ.2017.2762292

Copyright Institute of Electrical and Electronics Engineers

Additional Information 


\title{
Terahertz Sieves
}

\author{
Federico Machado ${ }^{1}$, Przemysław Zagrajek ${ }^{2}$, Juan A. Monsoriu ${ }^{1}$, and Walter D. Furlan ${ }^{3}$
}

\begin{abstract}
Imaging at terahertz (THz) frequencies offers a great potential for applications including: security screening, telecommunications biodetection, and spectroscopy. Some of these applications need specially designed lenses with customized characteristics that are not commercially available. In this work we present the THz sieves as a new kind of THz lenses. We demonstrate that these lenses improve the resolution of conventional zone plates constructed with the same level of detail. Amplitude and phase THz sieves were 3-D printed and tested experimentally. Excellent agreement was obtained between the experimental and calculated results.
\end{abstract}

Index Terms - Terahertz materials, Photon sieves, Terahertz focusing, and imaging.

\section{INTRODUCTION}

$\mathrm{N}$ UMEROUS applications of $\mathrm{THz}$ an sub- $\mathrm{THz}$ radiation such as imaging or spectroscopy require passive devices like lenses [1], filters [2], and waveguides [3]. Focusing lenses allow improving the sensitivity of terahertz setups, which is a crucial issue in this range of electromagnetic radiation, where there is a lack of high-power sources and high-sensitive detectors.

The optical properties of different polymers [4] have been exploited to fabricate a variety of $\mathrm{THz}$ lenses that are commercially available. However, many applications require custom-made $\mathrm{THz}$ lenses with a special design. Refractive lenses with different geometries have been manufactured from bulk polymers by lathe turning, compression molding [1], and recently, by $3 \mathrm{D}$ printing $[5,6]$. On the other hand diffractive $\mathrm{THz}$ optical elements have been proposed and tested $[7,8]$. In spite of their lower light throughput and chromatic aberration, diffractive lenses can have high numerical aperture [9], and permit beam shaping [10,11], working in linear and compact setups. Therefore, in many applications, the performance of diffractive $\mathrm{THz}$ lenses is better than that of their refractive homologous. Additionally, 3D printing technology has also been used recently to construct special designs of diffractive lenses [10-12].

Photon sieves (PSs) are diffractive optical elements,

Submitted October 02, 2017

This study was supported by the Ministerio de Economía y Competitividad and FEDER (Grant DPI2015-71256-R), Spain, and by the Generalitat Valenciana, (Grant PROMETEO II-2014-072), Spain. Partial support by the National Center for Research and Development in Poland (Grant LIDER/020/319/L-5/13/NCBR/2014) is also acknowledged.

${ }^{1}$ F. Machado and J. A. Monsoriu are with Centro de Tecnologías Físicas, Universitat Politècnica de València, Camino de Vera s/n, 46020 Valencia

${ }^{2} \mathrm{P}$. Zagrajek is with Institute of Optoelectronics, Military University of Technology, Warsaw, Poland

${ }^{3}$ W. D. Furlan is with Departamento de Óptica y Optometría y Ciencias de la Visión, Universitat de València, E-46100 Burjassot, Spain (email:walter.furlan@uv.es). originally conceived to improve X-rays focusing [13]. The first PSs where basically amplitude Fresnel zone plates (FZPs) in which the transparent rings were substituted by nonoverlapping holes of different sizes. Special features of PSs in the visible range were investigated in several works [14-17], from which different applications emerged [18,19]. The main features that characterize PSs are the following: (1) They can be fabricated on a single sheet without any substrate, (2) PS allow a better resolution than a Fresnel zone plate (FZP), with the same dimensions[13], (3) they allow improved focusing by the suppression of secondary maxima and higher orders of diffraction[16,17].

In this work, we introduce the $\mathrm{THz}$ sieves (TS). We study the TS axial and transverse resolution in comparison with those provided by a conventional FZP constructed with the same level of detail. The focusing properties of both, amplitude and phase TS are experimentally demonstrated.

\section{TERAHERTZ SIEVES. DESIGN AND FOCUSING PROPERTIES}

The construction procedure of a TS starts from a conventional FZP designed for a given $\mathrm{THz}$ frequency. As it is well known, an amplitude FZP of focal length $f$ at wavelength $\lambda$, consists of alternate transparent and opaque zones, where the radius of the $n$-th zone is given by $r_{\mathrm{n}}{ }^{2}=2 n f \lambda+n^{2} \lambda^{2}$.

The width of outermost ring of a zone plate with $N$ zones, $w=r_{N}-r_{N-1}$ (see Fig. 1a), imposes a limit on the maximum resolution achievable with the FZP: $w=\lambda f / 2 r_{\mathrm{N}}$. It was shown [13] that a PS can overcome this limitation because the pinhole diameter can be bigger than the width of the underlying zone. Thus a PS can have a higher numerical aperture than a FZP constructed with the same level of detail. This property is of particular interest in $\mathrm{THz}$ applications, where low-cost diffractive lenses can be $3 \mathrm{D}$ printed.

A TS is constructed by replacing the transparent rings of width $w$ in the Fresnel zone plate by non-overlapping circular holes of diameter $d$ distributed about the rings (see Fig. 1b). Thus, the transmittance function $t(x, y)$ of a TS can be expressed as a binary function that takes the values $t(x, y)=1$ if $\left(x-x_{i, j}\right)^{2}+\left(y-y_{i, j}\right)^{2} \leq d_{i, j}{ }^{2} ;$ and either $t(x, y)=0$ or $t(x, y)=-1$, otherwise; depending on if the TS is of amplitude or phase, respectively. In the transmittance function, $x_{\mathrm{i}, \mathrm{j}}$ and $y_{i, j}$ are the center coordinates of the holes, with $i=1,2, \ldots \mathrm{N}$ and $j=1,2, \ldots m$; being $m$ the number of holes in each zone.

The focusing properties of our proposal were assessed in comparison with a FZP by means of the irradiance at different planes for a point object at infinity i.e.; the point spread function (PSF). This function was computed numerically by using the nonparaxial scalar diffraction theory: 


$$
\begin{aligned}
& I(x, y ; z)=1 / \lambda^{2} \\
& \quad \times\left|\iint t\left(x_{0}, y_{0}\right) \frac{\exp \left\{i \frac{2 \pi}{\lambda} \sqrt{\left(x-x_{0}\right)^{2}+\left(y-y_{0}\right)^{2}+z^{2}}\right\}}{\sqrt{\left(x-x_{0}\right)^{2}+\left(y-y_{0}\right)^{2}+z^{2}}} d x_{0} d y_{0}\right|^{2},(1)
\end{aligned}
$$

We employed Eq. (1) to compare the focusing properties of phase TS and FZP. In particular we analyzed two lenses of the same focal distance: $f=122 \mathrm{~mm}$, at the same design frequency: $v_{0}=0.625 \mathrm{THz}$. For the lens material we considered polyamide PA6, which has a refractive index $n=1.59$ at this frequency[10]. The radius of the FZP $\left(r_{\mathrm{N}}=18.8 \mathrm{~mm}\right)$, and its outermost width $(w=1.63 \mathrm{~mm})$, were both selected to be compatible with an experimental verification in our lab. For the TS the minimum hole diameter was fixed to be $d_{\min }=1.65 \mathrm{~mm}$; i.e.; approximately of the same size of $w$. The maximum hole diameter was $d_{\max }=4.11 \mathrm{~mm}$. The hole density in each zone was selected such as the transparent area in each TS zone must be at least a $75 \%$ of the whole Fresnel zone. With these parameters we used Eq. (1) to compute the axial irradiance provided by the TS for different values of $d$, in the range $1 w \leq d \leq 2 w$, obtaining that the value $d=1.33 w$ provided the best apodization of the third diffraction order focus. Thus, we found that with the same width of the outermost ring, the numerical aperture of the TS is 1.33 times higher than the FZP, being the TS radius $r_{\mathrm{N}}=25 \mathrm{~mm}$. The axial irradiances and transverse intensities at the focal plane, provided by both lenses are shown in Fig. 1c) and Fig. 1d), respectively. As can be seen in Figs. 1c) and 1d) both the axial and transverse resolution are better for the TS. The full width at half maximum (FWHM) of the axial irradiance peak provided by the TS is $18.8 \mathrm{~mm}$, which is 52\% lower than FWHM of the FZP. For the transverse resolution the FWHM at the focus of the TS is $1.31 \mathrm{~mm}$, and 1.77 for the FZP. The apodization of the third order focus at $z=40 \mathrm{~mm}$ can be clearly seen in Fig. 1c). The physical reason for this apodization is that the axial irradiance only depends on the angular average of the effective pupil along the radial coordinate [20], which in the case of TS is smoothed by effect of the holes.

In order to investigate the effect of a finite bandwidth on TS performance, we have computed the axial PSF for the TS at two other frequencies. The result is shown in Fig. 2). In this case in addition to the focal shift produced by the chromatic aberration of the TS, the peak intensity is lower for the other two frequencies because for these frequencies the phase difference between the holes and the plate is not exactly $\pi$.
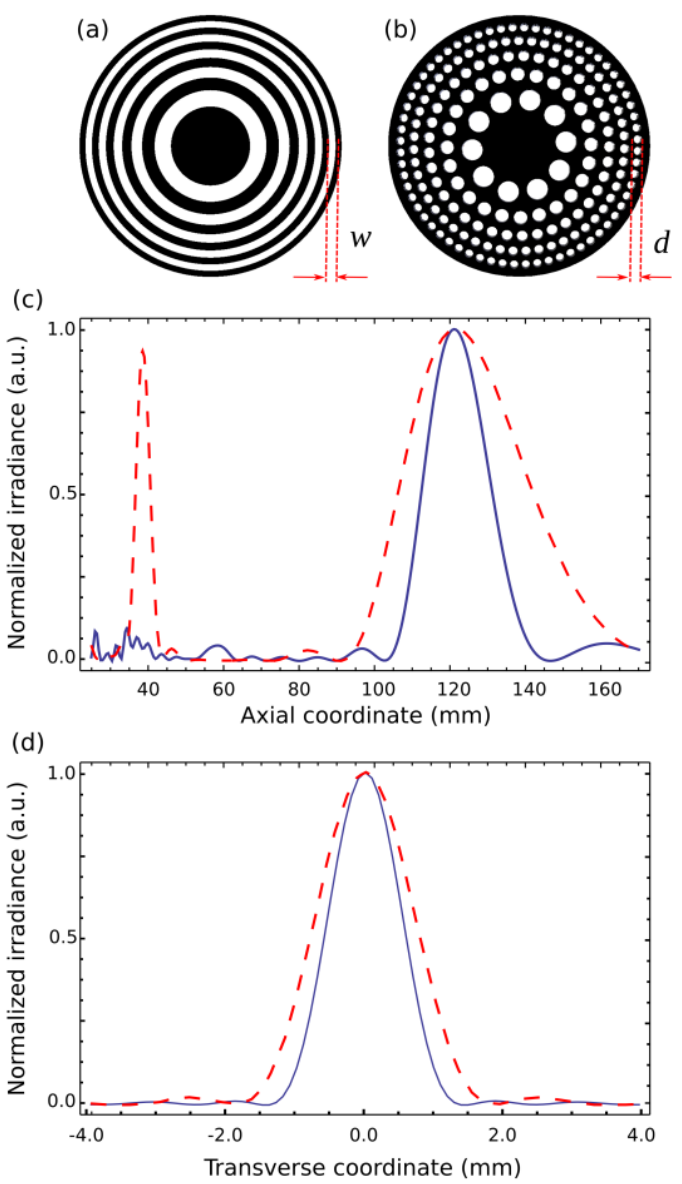

Fig. 1. a) Binary Fresnel zone plate, $w$ is the width of the outermost zone, which determines its transverse resolution. b) $\mathrm{THz}$ sieve with the same number of Fresnel zones, $d$ is the diameter of the holes at the Fresnel zone corresponding to $w$. c) Numerical axial point spread function; and d) Transverse Intensity at the focal plane, computed for a FZP (dashed line) and a TS (continuous line) of the same focal distance ( $f=122 \mathrm{~mm} ; v_{0}=0.625 \mathrm{THz}$ ). In Fig. 1c) and Fig. 1d) each plot was normalized to its maximum value

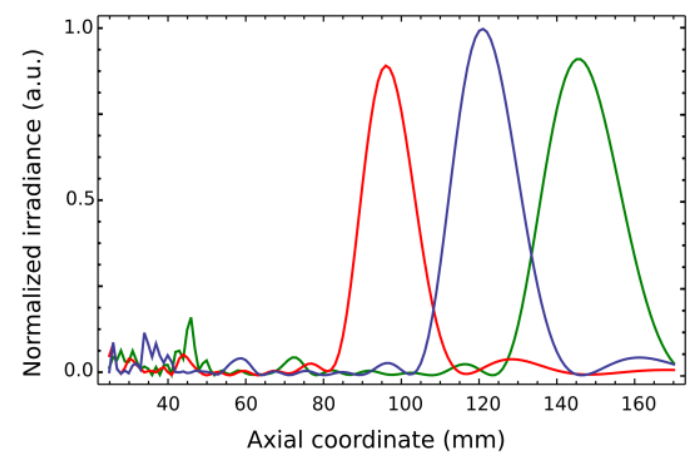

Fig.2. d) TS performance at 3 different frequencies: $v_{0}=0.625 \mathrm{THz}$ (blue line); $v_{1}=0.496 \mathrm{THz}$ (red line) and $v_{2}=0.741 \mathrm{THz}$ (green line). The maximum value of the irradiance for the design frequency was used for normalization. 


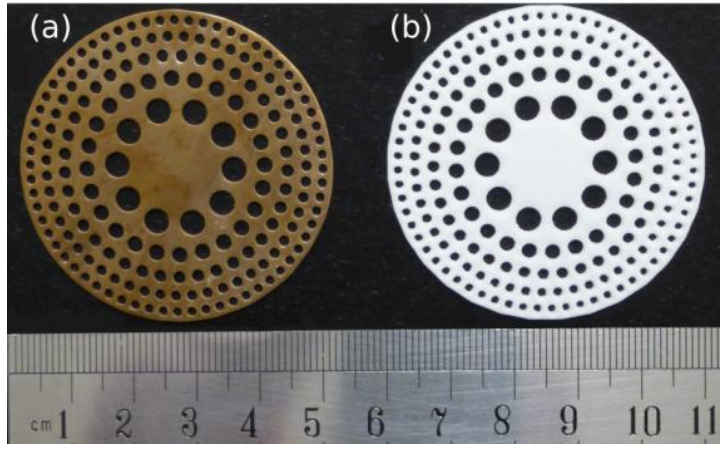

Fig.3. 3D printed $\mathrm{THz}$ sieves. a) Is an amplitude lens made in bronze. b) Is a phase lens made in PA6 polyamide. The holes are located in the even zones of a Fresnel zone plate (focal distance $f=122 \mathrm{~mm}$ ).

\section{EXPERIMENTAL RESULTS}

Fig. 4 shows the setup we employed for the experimental characterization of the TSs. A VDI frequency multiplier (Virginia Diodes, Inc. Charlottesville, VA. USA) provided the $0.625 \mathrm{THz}$ beam. The source of microwave radiation for the VDI frequency multiplier was an electromagnetic YIG-tuned oscillator MLXB-1768PA. It generates radiation in frequency range 13-15 GHz. The base frequency was multiplied 48 times. The divergent beam, emerging from the horn antenna, was collimated by a high density polyethylene (HDPE) lens and directed to the investigated TS. The detector: a Schottky diode, was mounted on a 3D motorized stage. The focal spot was scanned with a horn antenna, having a $2.4 \mathrm{~mm}$ aperture diameter. A lock-in system, based on modulation at $187 \mathrm{~Hz}$, and a mechanical chopper, was employed to measure the signal from the detector. The inset in Fig 4 shows the intensity recorded at the transverse plane $(x, y)$ just before the lens plane.

Two different TS lenses were fabricated by 3D printing: an amplitude lens, shown in Fig. 3a), constructed in bronze, and a phase lens, shown in Fig 3b), made in PA 6 polyamide. The physical dimensions of both lenses were the same, and coincide with those used to compute Fig. 1c) and Fig. 1d). As the minimum thickness of the phase TS that provides a $\pi$ phase difference between the lenses material and the holes $\mathrm{t}=\lambda / 2(\mathrm{n}-1)=0.4 \mathrm{~mm}$, was considered too thin to be handled, we have constructed the lenses with a thickness of $3 \mathrm{t}=1,2 \mathrm{~mm}$, which produces the same phase shift. Other details of the 3D lens production can be found elsewhere [10].

Fig. 5 shows the experimental results obtained for the axial PSF (dotted lines) corresponding to the lenses shown in Fig. 3. The numerical simulations obtained with Eq. (1) are shown in the same figure for comparison (continuous lines). To obtain the numerical results, the transmittance of the lens was multiplied by the normalized field amplitude recorded of the at the lens plane $(x, y)$ shown in Fig. 4). In addition, we not considered a point-like detector as in Fig. 1c), but integrated the irradiance in the area of detection at the horn antenna. Note that, in spite of that, the surface roughness, and microstructure of the $3 \mathrm{D}$ printed lenses have not been considered, we found a very good agreement between theory and experiment.

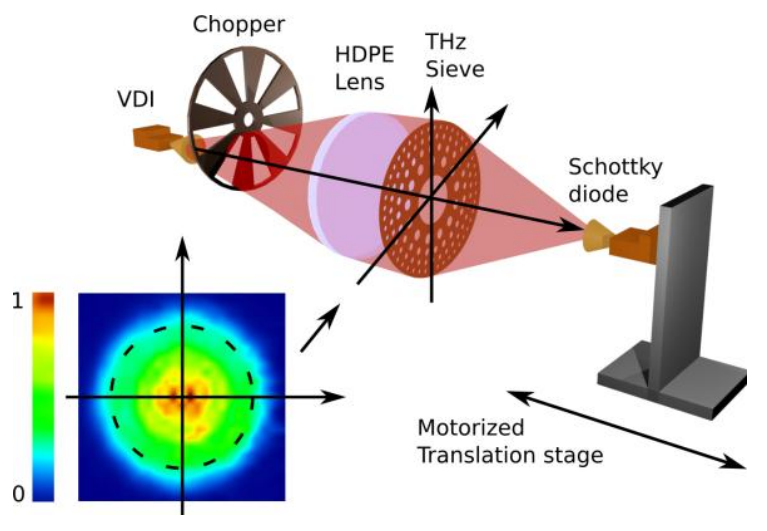

Fig.4. Experimental setup used in axial PSF measurements. The source of $\mathrm{THz}$ is a VDI frequency multiplier with a horn antenna. The divergent beam, is collimated by a HDPE refractive lens (focal distance $f=150 \mathrm{~mm}$ ). The transverse intensity measured at TS plane $(x, y)$ is shown in the inset. The radius of the dashed line in the inset is $r_{\mathrm{N}}=18.8 \mathrm{~mm}$. The detector: a Schottky diode, with a horn antenna, mounted on a 3D motorized stage, scanned the focal volume. A lock-in system, based on modulation at $187 \mathrm{~Hz}$, and a mechanical chopper, was employed to measure the signal from the detector.

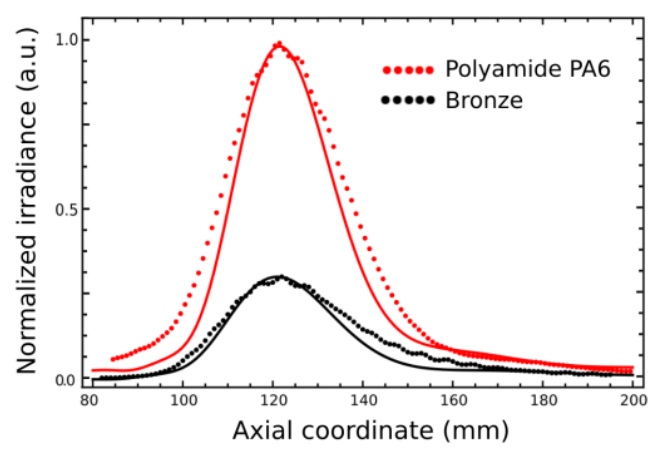

Fig.5 Experimental (dotted lines) and numerical simulation (continuous lines) provided by the 3D printed THz lenses of Fig. 3a) (black lines) and 3b) (red lines). The maximum value of the irradiance for the PA6 lens was used for normalization.

As expected phase polyamide TS is four times more efficient than the amplitude bronze TS of the same dimensions.

\section{CONCLUSION}

By using 3D printing technology, we have demonstrated the feasibility of realizing TS diffractive lenses. The focusing properties of both: amplitude and phase TS were tested using $0.625 \mathrm{THz}$ beam. We have shown that TS can achieve better resolution than binary FZP. Further improvements are expected with other exotic sieves distributions $[15,16]$. So, our proposal opens the possibility to use low cost optics for a wide range of $\mathrm{THz}$ applications. Two examples of special interest are $\mathrm{THz}$ astronomical telescopes where ultralarge space telescope primaries are necessary, preferably as a single membrane; i.e; with no supporting structure $[19,21]$ and $\mathrm{THz}$ compressive sensing, to achieve the precise focalization needed in a single pixel camera [22]. 


\section{REFERENCES}

[1] B. Scherger, M. Scheller, C. Jansen, M. Koch, and K. Wiesauer "Terahertz lenses made by compression molding of micropowders", Appl. Opt. vol. 50, no. 15, pp. 2256-2262, May. 2011.

[2] R. Wilk, N. Vieweg, O. Kopschinski, and M. Koch, "Liquid crystal based electrically switchable Bragg structure for $\mathrm{THz}$ waves," Opt. Express vol.17, no. 9, pp. 7377-7382, Apr. 2009.

[3] M. D’Auria, W.J. Otter, J. Hazell, B.T. Gillatt, C. Long-Collins, N.M. Ridler, and S. Lucyszyn. "3-D printed metal-pipe rectangular waveguides," IEEE Trans. Compon. Packag. Manuf. Technol., vol. 5, no. 9, pp.1339-1349. Sep. 2015.

[4] A. Podzorov and G. Gallot, "Low-loss polymers for terahertz applications," Appl. Opt. Vol. 47, no. 18, pp. 3254-3257, Jun. 2008.

[5] S. F. Busch, M. Weidenbach, M. Fey, F. Schäfer, T. Probst, and M. Koch, "Optical properties of 3D printable plastics in the $\mathrm{THz}$ regime and their application for 3D printed $\mathrm{THz}$ optics," J. Infrared Milli. $\mathrm{THz}$. Waves, vol. 35, no. 12, pp. 993-997, Dec. 2014.

[6] A. D. Squires, E. Constable, and A. Lewis, "3D Printed Terahertz Diffraction Gratings And Lenses," J. Infrared Millim. THz Waves, vol 36, no.1, pp.72-80, Jan. 2015.

[7] S. Wang, T. Yuan, E. Walsby, R. J. Blaikie, S. M. Durbin, D. Cumming, J. Xu, and X.-C. Zhang, "Characterization of T-ray binary lenses," Opt. Lett. vol. 27, no. 13, pp. 1183-1185, Jul. 2002.

[8] Y. Yu and W. Dou, "Generation of pseudo-Bessel beams at $\mathrm{THz}$ frequencies by use of binary axicons," Opt. Express, vol.17, no. 2, pp. 888-893, Jan. 2009.

[9] A. Siemion, M. Makowski, J. Suszek, J. Bomba, A. Czerwiński, F. Garet, J. Coutaz, and M. Sypek, "Diffractive paper lens for terahertz optics," Opt Lett, vol. 37, no. 20, pp. 4320-4322. Oct. 2012.

[10] W.D. Furlan, V. Ferrando, J.A. Monsoriu. P. Zagrajek, E. Czerwińska, and M. Szustakowski, "3D printed diffractive terahertz lenses," Opt. Lett. vol 41, no. 8, pp.1748-1751, Apr. 2016.

[11] C. Liu, L. Niu, K. Wang, and J. Liu. "3D-printed diffractive elements induced accelerating terahertz Airy beam," Opt. Express, vol. 24, no. 25, pp. 29342-29348. Dec. 2016.

[12] J. Suszek, A. Siemion, M.S. Bieda, N. Blocki, D. Coquillat, G. Cywinski, and A. Sobczyk, "3-D-printed flat optics for THz linear scanners," IEEE Trans. THz Sci. Technol. vol. 5, no. 2, pp. 314-316, Mar. 2015.

[13] L. Kipp, M. Skibowski, R. L. Johnson, R. Berndt, R. Adelung, S. Harm, and R. Seemann, "Sharper images by focusing soft x-rays with photon sieves," Nature, vol. 414, pp. 184-188, Nov. 2001

[14] Q. Cao and J. Jahns, "Nonparaxial model for the focusing of highnumerical-aperture photon sieves," J. Opt. Soc. Am. A, vol. 20, no. 6, pp. 1005-1012, Jun. 2003.

[15] F. Giménez, J.A. Monsoriu, W.D. Furlan and A. Pons, "Fractal photon sieve," Opt. Express, vol. 14, no. 25, pp. 11958-11963, Oct. 2006.

[16] F. Giménez, W.D. Furlan, and J.A. Monsoriu, "Lacunar fractal photon sieves," Opt. Commun. vol. 277, no. 1, pp. 1-4, Sep. 2007.

[17] A. Sabatyan and S. Mirzaie, "Efficiency-enhanced photon sieve using Gaussian/overlapping distribution of pinholes," Appl. Opt, vol. 50, no. 11, pp. 1517-1522, Apr. 2011.

[18] R. Menon, D. Gil, G. Barbastathis, and H. Smith, "Photon-sieve lithography," J. Opt. Soc. Am. A, vol. 22, no. 2, pp. 342-345, Feb. 2005.

[19] G. Andersen, "Large optical photon sieve," Opt. Lett. vol. 30, no. 22, pp. 2976-2978, Nov. 2005.

[20] C. J. R. Sheppard and Z. S. Hegedus, "Axial behavior of pupil-plane filters," J. Opt. Soc. Am. A 5, 643-647 (1988).

[21] S. Withington, "Terahertz astronomical telescopes and instrumentation," Phil. Trans. R. Soc. Lond. A, vol. 362, no. 1815, pp. 395-402. Feb. 2004.

[22] C. M. Watts, D. Shrekenhamer, J. Montoya, G. Lipworth, J Hunt, T.

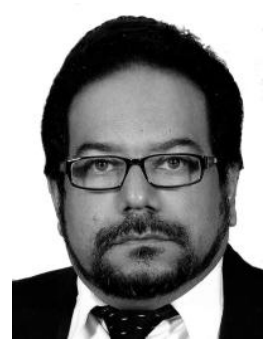
Sleasman, and W.J. Padilla, "Terahertz compressive imaging with metamaterial spatial light modulators," Nat. Photon., vol. 8, no. 8, pp. 605-609, Jun. 2014.

Federico Machado was born in El Salvador in 1970. He received the B.S. degree in mechanical engineering from
Universidad Politécnica de El Salvador in 1993 and M.S. degree on management of renewable resources from Universidad Don Bosco (El Stalvador) in 2013. He is currently pursuing the $\mathrm{Ph} . \mathrm{D}$. degree at Universidad Politécnica de Valencia. He worked at Universidad Don Bosco for 15 years in different positions, teaching subjects related to electrical, electronics and renewable resources. His experience is focused on energy efficiency and designing electronic systems to support educational systems.

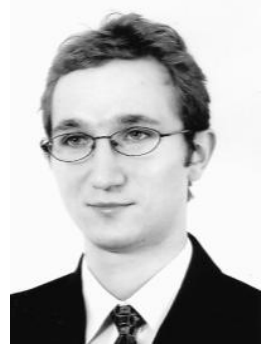

P. Zagrajek was born in 1979. He received his Master degree in Physics, at The Faculty of Applied Physics and Mathematics in Warsaw University of Technology in 2004 and Ph.D. degree in Institute of Physics, Polish Academy of Sciences. Warsaw, Poland in 2012. From 2012 he is an Assistant Professor with the Laboratory of Terahertz Technology at Institute of Optoelectronics at Military University of Technology, Warsaw, Poland. His areas of interest are spintronics and physics of nanodevices.

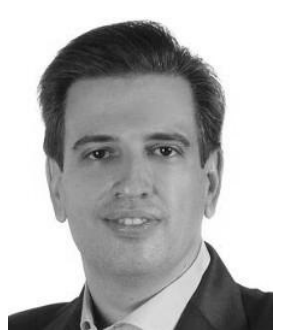

Juan A. Monsoriu was born in Valencia, Spain, in 1975. He received the B.S. degree in physics, M.S. degree in Optics, and Ph.D. degree in Physics from the Universidad de Valencia (UV), Spain, in 1998, 2000, and 2003, respectively. In 2000 he joined the Universidad Politécnica de Valencia (UPV), Spain, where he is currently Full Professor of Applied Physics. His research was performed at UV, UPV, Universidad de Málaga (Spain), University of Bath (UK), and Universidad de Buenos Aires (Argentina). His main research interests include numerical simulations for the design of microstructured optoelectronic systems and aperiodic optical devices.

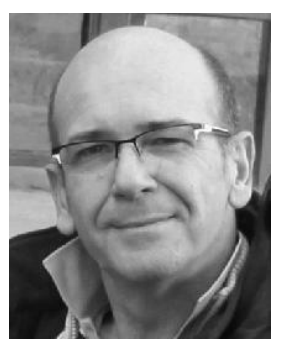

Walter D. Furlan received his MS and $\mathrm{Ph} . \mathrm{D}$. degrees in physics from the University of La Plata, Argentina, in 1984 and 1988, respectively. Until 1990 he worked at the Centro de Investigaciones Opticas (CIOp), Argentina. At the end of that year he joined the Optics Departament of the University of Valencia, Spain, where he is currently professor of Optics. His research has been developed in the field of optics in two well differentiated areas. On the one hand, he investigated on theory and applications of phase-space representations (Wigner Distribution Function, Ambiguity Function, etc.). In these topics he published more of 25 papers in refereed journals and a chapter in the book Phase-Space Optics: Fundamentals and Applications (McGraw Hill Professional, 2009). On the other hand, mainly in the last ten years, he focused on the study of 
> REPLACE THIS LINE WITH YOUR PAPER IDENTIFICATION NUMBER (DOUBLE-CLICK HERE TO EDIT)

the properties of non-conventional diffractive optical elements. He co-authored more than 30 papers and 3 patents related with these topics. Currently he is Full Professor at the University of Valencia, and co-director of the Diffractive Optics Group (DiOG). Dr. Furlan is a member of the European Optical Society. 
> REPLACE THIS LINE WITH YOUR PAPER IDENTIFICATION NUMBER (DOUBLE-CLICK HERE TO EDIT) 